Archives of Clinical and Medical Case Reports

doi: $10.26502 /$ acmcr. 96550053

\title{
Hemorrhagic Shock Status in ZAGAZIG Maternity University Hospital: Prognosis and Risk Factors
}

\author{
Khaled Fathy Helal, Mohamed El Sayed Fahmy* \\ Department of obstetrics and gynecology, Zagazig University, Zagazig, Egypt \\ "Corresponding Author: Dr. Mohamed El Sayed Fahmy, Department of Obstetrics and Gynecology, Zagazig \\ University, Zagazig, Egypt, E-mail: abrehab2000@yahoo.com
}

Received: 22 November 2018; Accepted: 12 December 2018; Published: 08 January 2019

\begin{abstract}
Objective: to assess the frequency and prognosis of hemorrhagic shock states in obstetrics in ZAGAZIG maternity university hospital.
\end{abstract}

Methods: a prospective study with descriptive and analytical purpose, carried out from January 1 to September 30, 2018 in ZAGAZIG maternity university hospital.

Results: We registered 1944 obstetrical emergencies. Among those emergencies, hemorrhagic shock status was diagnosed in 61 female patients, i.e. a hospital-based frequency estimated at 3.1\%. Patients' mean age was $27.0 \pm$ 6.3 years. $26.2 \%$ of those patients were between 20 and 24 years old. The mortality rate of hemorrhagic shock was $11.5 \%$. All causes combined, if we consider the 16 deaths registered in the medical unit during the same time period, the share of mortality associated with hemorrhagic shock was $43.75 \%$. After adjustment, the risk factors significantly associated with maternal death cases were uterine rupture $(\mathrm{p}=0.0000)$, coagulation disorders $(\mathrm{p}=0.0000)$ and Glasgow score below $9(\mathrm{p}=0.0285)$.

Conclusion: Obstetrical hemorrhagic shock was common in ZAGAZIG maternity university hospital. Its lethality is very high despite multidisciplinary management, hence the importance of early and adequate management of hemorrhage. 
Keywords: Hemorrhagic shock; Maternal mortality; Prognosis

\section{Introduction}

Reduction of maternal mortality is a priority goal in reproduction health, especially in developing countries. As a matter of fact, according to the World Health Organization (WHO), 850 women die every day across the world due to a cause associated with pregnancy, childbirth or post-partum. The larger part of this death toll i.e. $99 \%$ is registered in developing countries [1]. There is a large disparity in maternal mortality rates between northern and southern countries i.e.: 239 for 100000 births in developing countries versus 12 for 100000 births in developed countries. In more than $80 \%$ of cases, maternal mortality is due to hemorrhages, including $95 \%$ which would be preventable according to WHO. According to a study conducted in ZAGAZIG maternity university hospital in 2013, maternal mortality rate was estimated at 400 for 100000 and hemorrhagic emergencies came in second position (21.7\%) after dystocia (32.1\%) [2]. Obstetrical hemorrhage may rapidly develop into hemorrhagic shock which is life-threatening for woman. Hemorrhagic shock results in tissue hypoxia and organ dysfunction and pain [3]. Its management is based on simultaneous treatment of bleeding cause and consequences, particularly in terms of hemodynamic status and hemostasis $[4,5,6]$. Its management in developing countries is made difficult by sudden character of the shock, shortage or unavailability of intensive care resources (blood and blood derivatives), lack of skilled health workforce and populations' low socioeconomic status. Therefore, we decided to investigate the frequency, prognosis and risk factors for hemorrhagic shock status in the CHUDB/A maternity.

\section{Framework and Methods of Study}

This study was carried out in the maternity ZAGAZIG maternity university hospital, a referral hospital located in egypt.It was a prospective study with descriptive and analytical purpose, conducted during a time period of 9 months i.e. from January 1 to September 30, 2018. The study population consisted of all the women admitted to the emergency division of the ZAGAZIG maternity university hospital and presenting with hemorrhagic shock status to be addressed as obstetrical emergency. Our investigative work involved any woman in pregnancy or in postpartum phase presenting with hemorrhagic shock state associated with an obstetrical cause. Shock had been defined as heart rate higher than 100 beats per minute, blood pressure lower than or equal to $100 / 60 \mathrm{mmHg}$, respiratory rate osculating between 20 and 30 cycles per minute, moderate anxiety and thirst.

Our research work had not retained the cases of hemorrhagic shock status outside pregnancy or postpartum, and the cases of shock that were not caused by hemorrhage. We made an exhaustive recruitment of patients who meet study criteria. The study variables were sociodemographic variables, medical history, variables related to patient clinical condition, and variables regarding bleeding etiologies. Where this was possible, the data were gathered through a structured individual interview with women included in our study, after lifting of emergency and review of health booklet and medical record. Data collection was progressively done during the study time period. At each entry of one patient meeting the inclusion criteria, we engaged the procedure of monitoring and collection of the different information till permission to leave. 
We proceeded to a double entry of data using EpiData 3.1fr software. Data analysis and processing were done using SPSS (Statistical Package for the Social Sciences) software version 21. Descriptive statistics were generated for study variables. The quantitative descriptive variables were expressed as average \pm standard deviation when distribution is normal and the qualitative ones as number of subjects (size) and percentage. Fisher's exact test was used to compare frequencies. The difference existing between comparisons was considered as significant for any $\mathrm{p}$ value $<0.05$. We got an administrative authorization from the ZAGAZIG maternity university hospital Management and the maternity Head before starting this study. We complied with confidentiality and anonymity rules.

\section{Results}

\subsection{Shock status frequency and patients' mean age}

From January 1 to September 30, 2018 we registered 1944 obstetrical emergencies. Among those emergencies, hemorrhagic shock status was diagnosed in 61 patients, i.e. a hospital-based frequency estimated at 3.1\%. Patients' mean age was $27.0 \pm 6.3$ years. The age of $26.2 \%$ of those patients oscillated between 20 and 24 years.

\subsection{Prognosis of shock status}

2.2.1. Mortality: frequency and causes: Among the 61 cases of hemorrhagic shock, 7 died i.e. a lethality of $11.5 \%$.

All causes combined, if we consider the 16 deaths registered in the medical ward during the same time period, the share of mortality associated with hemorrhagic shock was $43.75 \%$ (7/16). The causes of death were delay in referral (4 deaths) and delay in care provision (3 deaths) due to shortage of blood products.

\subsubsection{Factors associated with death:}

2.2.2.1 Sociodemographic features: Deaths were registered in patients aged 30-34 years (20\%) and in those aged 35 years or more (33.3\%).There was no statistically Signiant relationship between place of residence, educational background and mortality. The distribution of death cases according to sociodemographic features is summarized in Table 1.

2.2.2.2 Data of the test done on admission: Among the patients with Glasgow score below 9, five (5) died (23.8\%) versus 2 deaths (5.0\%) among patients who had Glasgow score above or equal to 9. Glasgow score was statistically associated with mortality $(\mathrm{p}=0.028$ ), even after adjustment. The same applied to coagulation disorders. The distribution of death cases based on data of the test done on admission is synthetized in Table 2 .

2.2.2.3 Etiology: There was a significant association between ruptured uterus and death of patients $(\mathrm{p}=0.000)$. Coagulation disorders were significantly associated with patients' mortality $(\mathrm{p}=0.004)$. The distribution of death cases according to causes of hemorrhagic shock is synthetized in Table 3. 
2.2.2.4 Risk factors after adjustment: After adjustment, the risk factors significantly associated with maternal death are rupture of uterus $(\mathrm{p}=0.0000)$, coagulation disorders $(\mathrm{p}=0.0000)$ and Glasgow score below $9(\mathrm{p}=0.0285)$. The final model of multivariate analysis of risk factors after adjustment is summarized in Table 4.

\section{Discussion}

In our research work, frequency of hemorrhagic shock status is estimated $3.1 \%$ of patients hospitalized in the ZAGAZIG maternity university hospital [7] had found a ratio of 0.4 death for 100 childbirths in Morrocco. The incidence of hemorrhagic shock reported in literature is estimated at $1 \%$ of pregnancies according to Cohen [8], a ratio which is lower than our frequency. Other authors have found out frequencies higher than ours; for instance, Harrison KA et al. [9] had found $6.1 \%$ in a study conducted in Nigeria and Chattopadhay SK et al. [10] 5.2\% of hospitalizations for gynecological and obstetrical reasons. The high frequency of hemorrhagic shock noted in our cohort may be due to the fact that the CHUDB/A is the largest referral hospital in the Borgou/Alibori region; so, it is the last resort for many patients.

We registered 7 deaths out of 61 cases of shock state i.e. a lethality of $11.5 \%$. This ratio is higher than the one obtained by Foumsou et al in Chad and by Chaoki et al in Tunisia [11, 12] who report respectively 3.7\% and $1.4 \%$ of lethality due to hemorrhagic shock. This high level of lethality is partially attributable to lack of blood products, lack or poor readiness of patients before their referral and transportation which often is not medicalized. For instance, most of the health centers referring patients had no ambulance. Most evacuations or referrals were implemented by means of taxis, personal cars and motobikes. Besides, among the 16 deaths registered in the ward during the study time period, hemorrhagic shock was cause of death in 7 patients i.e. a mortality rate of $43.75 \%$. This rate is higher than the ones reported respectively by Arrandani A in Morrocco estimated at 35\% [13] and Traoré B et al in Mali who have reported 38.4\% [14]. In France, maternal mortality due to hemorrhages is estimated at $19.1 \%$ [15]. Therefore, obstetrical hemorrhages remain one of the leading causes of maternal mortality across the world, particularly in developing countries. As regards associated factors after adjustment, three factors proved to be significantly associated with maternal mortality. These are uterine rupture, coagulation disorders and Glasgow score below 9. Those factors are actually the outcome of several factors which will result in the weakening and rupture of the uterus, massive blood loss till onset of coagulation disorders, and in multiple organ failure, thus leading, amongst other consequences, to altered state of consciousness.

According to many researchers, advanced age is a significant prognostic factor even though it did not seem more significant after adjustment in our study. However, all cases of death were registered in patients aged 30 years and above in our study. Risk for maternal mortality is correlated with age according to many authors. For Bouvier et al, that risk is minimum between 20 and 24 years and remains low till 29 years. It increases significantly as from 35 years since it is three times higher than from 35 to 39 years and twelve times higher at 45 years compared with group of patients aged 20 to 24 years [16]. In the cohort of Fenomanana in Madagascar in 2008, age above 35 years represents 12.2 times more risk for mortality as outcome of postpartum hemorrhage compared with group of patients 
aged 18 to 34 years [17]. This is due to the fact when the subject gets older, other risk factors appear e.g. multiparty. Therefore, those women accumulate risks associated with age and those associated with multiparty. In our cohort, 7 deaths were registered among multiparous women, even though this factor does not appear as more significant after adjustment. Lethality was estimated at 55.6\% among patients with ruptured uterine, and at $75 \%$ in those presenting with a coagulation disorder.

There was a statistically significant association between mortality of hemorrhagic shock and uterine rupture $(\mathrm{p}=0.000)$. Among the women with unscarred uterus, that rupture is not common, but is in general complete and severe. The situations associated with occurrence of uterine rupture are previous uterine surgery, direct or indirect genital trauma, inappropriate use of oxytocin and grand multiparty. The therapy principle of uterine rupture is correction of hemorrhagic shock and emergency response of surgical hemostasis to reduce the frequency of mortality. However, the lack of quick and coordinated management is a common reality.

Hemodynamic status had a significant prognostic value. Unfortunately, more often patients arrive at a time when constants were already seriously altered, thus reflecting a deep shock status. Therefore, those patients often arrive in an irreversible shock state with coagulation disorders. This makes resuscitation endeavors practically useless. This may be due to a poor monitoring of newly delivered women and women who were bleeding, by either ignorance or negligence. In our cohort, high mortality has been registered among patients with unmeasurable blood pressure as well as thread and unmeasurable pulse. Six out of seven deaths registered occurred among the 10 patients with unmeasurable pulse $(60 \%)$. There was also a statistically significant association between hemorrhagic shock lethality and coagulation disorders $(\mathrm{p}=0.004)$. For Bennani H (Morrocco in 2002), appearance of hemodynamic failure with systoloic blood pressure below $60 \mathrm{mmhg}$ and onset of hemostasis disorders compromise maternal survival from hemorrhagic shock states [18].

\section{Conclusion}

Hemorrhagic shock status is an obstetrical complication which is serious and common in developing countries. Hemorrhagic shock may occur at any time of pregnancy. Its early diagnosis as well as its quick and coordinated management should help improve its prognosis.

\section{References}

1. Organisation Mondiale de la Santé (OMS). Mortalité maternelle. Centres des médias. Principaux faits, Genève OMS (2018).

2. Tchaou AB, Hounkponou MFN, Salifou K, et al. Obsterical emergencies in the university teaching hospital of clinical, therapeutic features and outcome. European Scientific Journal 11 (2015): 260-272.

3. Schadt JC, Ludbrook J. Hemodynamic and neurohumoral response to acute hypovolemia in conscious mammals. Am J Physiol 260 (1991): 305-318.

4. Fernandez H. Obstetrical hemorrhages. Human reproduction and hormones 8 (1995): 39-46. 
5. Goffinet F. Delivery hemorrhages. Gynecol Obst et Fertilité 28 (2000): 141-151.

6. Carroli G, Cuesta C, Abalos E. Epidemiology of postpartum haemorrhage: a systematic review. Best Pact Res Clin Obstet Gynecol 22 (2008): 999-1012.

7. Kodjo JAM. The management of peripartum hemorrhage in the intensive care unit: a review of 96 cases) [Thesis]. Sidi Mohamed Ben Abdallah University, Morocco (2013): 114.

8. Cohen W, Oliviennes F. Postpartum hemorrhages. A practitioner's review 45 (1995): 1777-1781.

9. Vanhoutte A. Gynecological and/or obstetrical hemorrhages. Urgence 16 (2009): 969-979.

10. Abboud FM. Ventricular syncope. Is the heart a sensory organ? N Engl J Med 9 (1989): 390-392.

11. Foumsou L, Mahamat P, Damtheou S, et al. Immédiate postpartum hemorrhages: epidemiological aspects and prognosis in the Mother \& Child Hospital of N'Djamena - Chad. AJIH 5 (2014): 14-17.

12. Chaouki M, Marwen N, Amjed A, et al. Severe postpartum hemorrhagie: epidemiology and management in the University Teaching Hospital of Nabeul, Tunisia. Ann. Afr. Med 6 (2013): 1-6.

13. Arramdani A. Maternal mortality: An experience from the mother \& child intensive care of the Hassan II University Teaching Hospial (CHU) of Fès (A review of 60 cases) [Thesis]. Sidi Mohammed University, Morrocco (2014): 130.

14. Traore B, Thera TA, Kokaina C, et al. Maternal mortality in the gynecology and obstetrics unit of the regional hospital of Segou (Mal)i. A case-control study of 138 cases. Mali médical 25 (2010): 42-47.

15. Bouvier-Colle MH, Deneux-Tharaux C, Aucedo M, et al. Maternal deaths in France: improving understanding for better prevention; 2007-2009 Report of the National Commitee of Experts on Maternal mortality. Legal deposit: November (2013).

16. Bouvier-Colle MH, Ouedraogo C, Dumont A. Maternal mortality in West Africa: rates, causes and substandard care from a prospective survey. Acta Obstet Gynecol Scand 80 (2001): 113-119.

17. Fenomanana MS, Riel AM, Rakotomena SD, et al. Risk factors for mortality due to postpartum hemorrhages in the Maternity of Befelatanana - University Teaching Hospial (CHU) of AntananarivoMadagascar. Rev Anest Réa Méd Urg 1 (2009): 4-7.

18. Bennani H. Obstetrical hemorrhagic shock. [Thesis]. Faculty of Medicine and Pharmacy, Morrocco (2002): 123.

Citation: Khaled Fathy Helal, Mohamed El Sayed Fahmy. Hemorrhagic Shock Status in ZAGAZIG Maternity University Hospital: Prognosis and Risk Factors. Archives of Clinical and Medical Case Reports 3 (2019): 510.

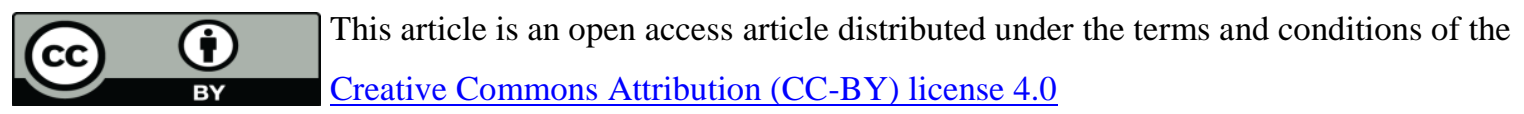

